\title{
La vaguedad de la vida: la Terceridad de Peirce en los sistemas biológicos ${ }^{*}$
}

\author{
The Vagueness of Life: Peirce's Thirdness in Biological Systems \\ José Agustín Mercado Reyes ${ }^{\dagger}$
}

\begin{abstract}
Resumen
La filosofía de Charles S. Peirce gira alrededor de un sistema triádico de categorías que expresa su postura metafísica acerca de la realidad. El presente texto realiza una interpretación de la categoría de Terceridad y de la noción de "vaguedad" asociada a ella, a la luz del conocimiento biológico actual. La aplicación de dicha categoría nos permite entender desde otra perspectiva el concepto de información biológica, ofreciendo una respuesta a las recientes críticas a dicho concepto: lejos de resultar una mera metáfora, la información se vuelve un elemento real y fundamental de los sistemas biológicos.
\end{abstract}

Palabras claves: información biológica - realismo escolástico - biosemiótica - Terceridad

\begin{abstract}
Charles S. Peirce's philosophical system turns around a triad of categories that underlies his metaphysical conception of reality. I offer an interpretation of the category of Thirdness, and of the closely related notion of "vagueness", in terms of our current understanding of biological systems. By looking at the concept of biological information through the lens of Thirdness, we can offer a rejoinder to the critics of said concept: far from being a mere metaphor, information becomes a real and fundamental element of biological systems.

Keywords: biological information - scholastic realism - biosemiotics - Thirdness
\end{abstract}

* Recibido: 10 de Junio de 2014. Aceptado en versión revisada: 30 de Agosto de 2014.

+ Doctorando del Posgrado en Filosofía de la Ciencia, UNAM, México. Para contactar al autor, por favor, escribir a: agustin_mr@ciencias.unam.mx.

* Trabajo realizado con apoyo de la beca de posgrado no. 358381 del Consejo Nacional de Ciencia y Tecnología, México. Metatheoria 5(1)(2014): 65-78. ISSN 1853-2322.

(C) Editorial de la Universidad Nacional de Tres de Febrero. Publicado en la República Argentina. 


\section{Introducción}

Una de las mayores dificultades de la obra de Charles Sanders Peirce es que prácticamente toda ella gira en torno a una gran idea. Esta idea no es caracterizable en una única palabra; su manifestación más clara es la noción compleja de realidad que Peirce desarrolló sistemáticamente en la mayor parte de sus escritos. El objetivo del presente texto es doble. En la primera parte, haré una lectura e interpretación de la categoría de Terceridad, el cual es uno de los conceptos centrales de dicha noción de la realidad. En la segunda, exploraré las consecuencias de aplicar este concepto al estudio de los sistemas biológicos. El argumento principal es sencillo: un recuento de la realidad en general y de los sistemas biológicos en particular resulta incompleto sin incluir los fenómenos que Peirce agrupó bajo el concepto de Terceridad. Si consideramos como real a la Terceridad, una multitud de conceptos utilizados en biología -incluida la noción de información, el ejemplo central del presente texto- escapa a las críticas de ser simples metáforas, sin referente y reificadas, y adquieren una vitalidad distinta.

La noción compleja de realidad que despliega Peirce alimenta constantemente su pensamiento, si bien en ocasiones de manera velada y subterránea. Desde mi punto de vista, un entendimiento superficial de sus conceptos en un área determinada (sea esta área su semiótica, o sus propuestas lógicas, o su peculiar sistema cosmológico) es irremediablemente incompleto sin considerar el intercambio que constantemente ocurre con su visión metafísica. Por lo tanto, el trayecto de este texto pasa por diversas estaciones antes de entrar de lleno al análisis biológico. Sólo después de discutir los argumentos de Peirce a favor de la necesidad de entender a la realidad a través de una serie tricotómica de categorías (sección 2), y de explicar las distintas instanciaciones y la evolución de su pensamiento que llevaron a la enunciación más general de estas categorías (sección 3), intento conectar una de las categorías, específicamente la indeterminación de la llamada Terceridad, (explorada en la sección 4) con el reciente debate acerca del concepto de información biológica (sección 5).

\section{Efectos en la realidad}

Durante el texto de "What Pragmatism Is" (Peirce 1992a), Peirce explica su sistema filosófico. Para tal fin, pone en escena una especie de catecismo e imagina a un interlocutor que cuestiona cada paso de su argumento y a quien contesta pacientemente. El interés del escrito, en tanto que trata de fundamentar la naturaleza del significado de proposiciones, es mostrar que una concepción (i.e., lo que racionalmente da a entender una palabra o una expresión) es coextensiva al efecto que tenga en alguna conducta. Textualmente: "Consider what effects that might conceivably have practical bearings you conceive the object of your conception to have. Then your conception of those effects is the WHOLE of your conception of the object" (Peirce 1992a, p. 338). Esta enunciación ya ha aparecido y seguirá apareciendo, exactamente igual, a través de muchos escritos de Peirce bajo el nombre de "la máxima del pragmatismo".

Hasta ese momento, Peirce nos parece llevar de la mano hacia una teoría especial del lenguaje, o dar un recuento psicológico de lo que significa un concepto, engarzado completamente en un interés discursivo. Desde cualquier punto de vista, Peirce se mueve en terrenos completamente antropocéntricos: la ciencia humana construye conceptos, y éstos tienen efectos sobre conductas, creencias y hábitos de las personas. Sin embargo, el intercambio entre interrogador y pragmatista ocurre en un crescendo que culmina en la última pregunta: "Well, if you choose so to make Doing the Be-all and the End-all of human life, why do you not make meaning to consist simply in doing?" (Peirce 1992a, p. 341). En este momento, Peirce deja

${ }^{1}$ Todas las referencias al trabajo de Peirce están dadas en dos ediciones: la Essential Peirce (EP) y Collected Papers (CP), seguidos del número de volumen y el número de página. 
atrás las explicaciones acerca de teorías del lenguaje; en vez de dar una respuesta, comienza a meditar acerca de la pregunta misma de su interlocutor. Es evidente que ha fracasado en explicar su visión filosófica a su crítico, quien sigue sin entender nada. Desde luego -responde Peirce- que el pragmatismo no se trata únicamente de hacer: el "hacer" tiene que "hacerse en un tiempo específico, sobre un objeto específico". Esta dimensión, que en otros textos es nombrada como hecceidad (haecceity), hace referencia a un tipo específico de determinación: la determinación de tiempo y espacio, el modo de ser que ocurre aquí y ahora (hic et nunc). Dicho en otras palabras, son eventos individuales o, en la terminología de Peirce, existentes. En contraste, los efectos de los conceptos tienen el carácter de generalidad.

Este salto aparentemente brusco, desde una discusión centrada en semiosis a una centrada en modos de ser, señala uno de los puntos de articulación de la filosofía peirceana. Peirce se niega a considerar a una realidad compuesta de planos completamente separados entre sí; lo que hay "aquí y ahora" convive con los efectos de los conceptos, y es parte importante de su trabajo determinar cómo y en qué condiciones ambos planos conviven. También se niega a escindir su sistema de cualquier tipo de manifestación que tenga efectos detectables, independientemente de la forma de detectarlos. En la visión de Peirce, hay elementos cuya realidad atestiguamos por sus efectos, y que no pueden ser descritos en su totalidad en un nivel de hecceidad; estos elementos pierden algo al ser tratados bajo un marco ontológico que sólo acepta entidades individuales. Desde luego, sería absurdo decir que las entidades individuales no tienen un lugar en la descripción de la realidad. Un auto que se impacta con otro es un penoso recordatorio de lo que la fuerza bruta, en un momento y un lugar determinado, puede lograr. Sin embargo, cuando escribo "un auto que se impacta con otro”, ¿de qué autos estoy hablando? ¿Son autos azules, rojos, alemanes o japoneses? ¿En dónde, cuándo y cómo se produce el impacto? ¿Cuál es específicamente el daño que se ha causado? Claramente, nada de eso está contenido en la frase que acabo de escribir; y sin embargo, la frase ha causado un efecto en el lector mínimamente atento: la imagen, clara o confusa, de un accidente automovilístico. Hay algo fundamentalmente distinto entre un evento individual (el choque entre dos autos) y una referencia general a un conjunto de estados del cual este tipo de evento individual puede formar parte (la frase "el choque entre dos autos").

Éste es precisamente el punto central del texto de "What Pragmatism Is" (Peirce 1992a): la realidad está compuesta no solamente por elementos que Peirce llama existentes, que están completamente determinados y que tienen un carácter de individualización. El mensaje principal de Peirce es el siguiente: cuando no podemos describir la totalidad de la realidad bajo un esquema ontológico, es decir, cuando se presentan ante nosotros instancias que requieren algo más para que podamos dar cuenta de ellas, debemos de tomar a este esquema como incompleto. Su búsqueda, desarrollada a lo largo de toda su vida, se centra en encontrar estos elementos faltantes, caracterizarlos y describirlos. A lo largo de su carrera profesional, informada simultáneamente por vertientes científicas y filosóficas, Peirce cree toparse una y otra vez con este déficit. Esta búsqueda de elementos faltantes se cristaliza en su sistema de categorías. ${ }^{2}$ La lista de categorías que Peirce deseaba hacer era una "lista corta", en la que cada una de las categorías fuera universal y necesaria, y no una "lista larga", en la que algún elemento de la realidad cualquiera pudiera presentar sólo algunas categorías y no otras (Hausman 1993, p. 95). Esto implica, como se verá más adelante, que en una lista corta sólo podemos hacer la separación de una de las categorías realizando una abstracción mental de las otras dos, a diferencia de las categorías de la lista larga, que no es exhaustiva y en la que cada categoría se puede tratar de manera completamente independiente a las demás.

Al considerar a las categorías como parte de la realidad, Peirce marca una dicotomía con la que clasifica cualquier postura filosófica. En un lado, la posición del propio Peirce, que llama "realismo escolástico". En el otro, los "nominalistas"; es decir cualquiera con quien Peirce tuviera alguna discrepancia: entre muchos

\footnotetext{
2 "A New List of Categories" (Peirce 1992b) de 1867 es una de las primeras publicaciones de Peirce, realizada cuando tenía sólo 27 años; el propio Peirce escribe en una carta de 1905 que toda su vida consideró a esa lista como "mi única aportación a la filosofía" (Peirce 1931, vol. 8, p. 213).
} 
otros, Descartes, Kant, Leibniz, Spinoza, Hobbes, Hume, Berkeley, Mill, e incluso William James y Duns Scotus, filósofos que Peirce consideraba los más cercanos a su propio pensamiento (Haack 1992). Aunque colocar a tantos y tan variados autores en un solo saco es claramente problemático, creo que podemos seguir la intención de Peirce en su texto "Seven Systems of Metaphysics" (Peirce 1992c): para ser nominalista, un sistema filosófico tiene que considerar al menos una de las categorías como fuera de la realidad. Esto puede ocurrir, por ejemplo, cuando pensamos que alguna categoría es un derivado ilusorio de nuestra cognición imperfecta, o cuando introducimos una barrera de inaccesibilidad en donde algo queda fuera de nuestro alcance (debido que para Peirce la realidad debe de diagnosticarse mediante los efectos que causa, según su máxima pragmática). Los "siete sistemas", pues, son las combinatorias metafísicas posibles: una que toma las tres categorías como reales, y seis posturas nominalistas y erróneas, que declaran como irreal una o más categorías.

\section{Avatares de las categorías}

El tejido de la realidad, nos indica Peirce, se compone de hebras de cualidades distintas. No se puede enfatizar lo suficiente que con esto Peirce no desea subvertir gratuitamente la experiencia diaria. Todo lo opuesto: diversas manifestaciones de la propia realidad revelan en sí una riqueza individual que va más allá de cualquier descripción que podamos hacer. Pensemos en el campo de estudio que está inevitablemente unido al nombre de Peirce, la semiótica. Un signo cualquiera tiene una infinidad de maneras de ocurrir; incluso podemos considerar que el desarrollo de un signo ha sido interrumpido, o que éste ha sido interpretado de manera distinta a la que se pretendía. Nos encontramos frente a un contraste entre (al menos) dos modos de comprender el fenómeno: hay una riqueza en el evento singular que escapa a cualquier descripción completa, y simultáneamente podemos catalogar a dicha ocurrencia singular como parte de un conjunto de efectos, potencialmente infinito, en el que puede reconocerse la acción de un signo.

Singular y general: esta especie de mirada doble sobre un mismo evento es una de las más básicas particiones en el recuento que Peirce da de la realidad (aunque como se verá, no es la única). Si aceptamos ambos lados de la partición, aceptamos por default un incremento de las entidades que componen a la realidad. Estamos, de hecho, multiplicando entidades. Peirce acepta precisamente en estos términos el reto de un adversario conceptual, William de Ockham. A pesar de ser el ejemplo primario de la posición contra la que escribe (baste decir que, en ocasiones, llama "ockhamistas" a los nominalistas), Peirce admira algunas prescripciones metodológicas del filósofo medieval (Boler 1980). En su famoso dictum, Ockham insiste que entia non sunt praeter multiplicanda necessitatem: la multiplicación de entidades sólo se puede llevar a cabo cuando es necesario. El desideratum es una especie de parsimonia ontológica, y por lo tanto aquel que quiera complicar la ontología debe de mostrar la verdadera necesidad de hacerlo.

Las pruebas que aporta a Peirce frente a la parsimonia son en un principio lógicas y centradas en un análisis proposicional. El punto de partida de esta concepción es la formalización de la lógica de las relaciones, en la que afirma que las relaciones irreducibles son exactamente tres. La primera, la relación monádica, en la que sólo existe un relatum en la relación, por lo cual se logra mantener únicamente con una referencia a sí misma. Llamada "cualidad" en esta primera iteración, la relación primera se presenta como una abstracción completa: por ejemplo, la cualidad abstracta de "blanqueidad”. La segunda relación, la diádica, comprende dos relata, y presupone la primera; el ejemplo típico está en la proposición "el escritorio es blanco": hay una referencia directa a una objeto existente, que se relaciona con las bases monádicas (que existen por sí mismas). Finalmente, la relación triádica, la representación, presupone tres relata en una relación irreducible. El ejemplo peirceano más conocido es la proposición que habla de regalar algo: X regala Y a Z. Si intentamos reducir esta relación a proposiciones diádicas ("X sostiene el objeto Y", "X suelta el objeto Y", "el objeto Y cae en la mano de Z", etc.), podemos complicar tanto como 
queramos, pero hay algo que se pierde de nuestra descripción: el acto mismo de regalar.

Para nuestro argumento actual, son irrelevantes las críticas que se pueden hacer, y de hecho se han realizado $^{3}$ al recuento de lógica triádica de Peirce. Lo que importa es que prepara el escenario para otro análisis triádico, que se centra en preocupaciones metafísicas. Hacia 1890, en su boceto para un libro inconcluso llamado "A Guess at the Riddle" (Peirce 1992d) Peirce comienza a liberar a sus categorías de los terrenos de la proposición, proponiéndolas como una manera de organizar relaciones a lo largo del espectro de la investigación científica, como él la entiende. El camino que se inicia con el análisis lógico alcanza su madurez en lo que Peirce llama "fenomenología" (en 1902) y, posteriormente, "faneroscopía" (1904). A pesar de la obvia coincidencia de nombre con la tradición que inicia Husserl, aparentemente Peirce desarrolla su teoría "fenomenológica" de manera independiente, centrándose en su concepto de phaneron: aquello que se presenta frente a la mente, independientemente de su existencia física. ${ }^{4}$

Al enfatizar el efecto que el phaneron tiene en una mente marca claramente el viraje que Peirce da a sus categorías inicialmente lógicas. De hecho, en su última década deja de lado las demostraciones en términos de relaciones abstractas (i.e., mónada, díada, tríada) y enfatiza la percepción de un fenómeno. Es importante notar que a pesar de hablar de mente o percepción, Peirce está alejado del psicologismo. Como un eco de la filosofía de Henri Bergson, quien justo en esos mismos años desarrollaba su visión metafísica centrada alrededor de sus conceptos de duración, simpatía e intuición (ver Bergson [1903]_2011), Peirce acepta que nuestra relación del mundo está limitada por nuestras posibilidades de percepción y pensamiento. Sin embargo, hay una serie de técnicas que podemos adoptar para esclarecer nuestras ideas. Por ejemplo, podemos mostrar una instancia tras otra de un concepto ajeno a nosotros y, mediante un acercamiento a lo que sí se presenta ante nuestra mente lograr una especie de iluminación, un acceso a otras características de las cosas en sí mismas.

En su instanciación fenomenológica, las categorías comienzan a ser mostradas con sus ejemplos típicos y mejor logrados. La relación monádica es definida como una posibilidad de sensación: una cualidad abstracta, no actualizada ni en hecho ni en pensamiento, separada de toda instanciación en tiempo o espacio. ¿Cómo podemos pensar una cualidad de este estilo, separada de cualquier recuerdo o ejemplificación? No podemos, contesta Peirce: "It cannot be articulately thought. Assert it, and it has lost its characteristic innocence. Stop to think of it, and it has flown!" Y un poco más adelante: "Every description of it must be false to it" (Peirce 1931, p. 1357).

Al pensar, recordar o instanciar la posibilidad monádica -es decir, al actualizarla de cualquier maneracaemos automáticamente en el "segundo universo", aquel de objetos existentes y los hechos sus interacciones brutas (Carta a Lady Welby [ver Peirce 1992e, p. 479]). El ejemplo típico es la lucha entre dos fuerzas. Imaginemos que intento abrir una puerta, y la puerta se resiste. Hay alguien o algo detrás de ella que me lo está impidiendo. La resolución viene en cuanto una de las fuerzas se impone sobre la otra, claro; pero la experiencia misma de sentir la fuerza que empuja y resiste resume el mundo de las relaciones diádicas. Acción/reacción: no hay manera de reducir esto a términos de relaciones monádicas, debido a que la resistencia presupone algo a lo cual resistirse.

¿Cómo se vería un universo en donde sólo esté en juego la Segundidad? Según Peirce, sería ininteligible. Los hechos brutos, por sí mismos, son inmediatos e instantáneos; un universo compuesto por Segundidad sería el caos absoluto, pues no habría conexión entre los hechos. La Representación (aquello que se presenta ante la mente como una tríada irreducible a relaciones de menos elementos) es ante todo la inclusión de una dimensión temporal en el recuento fenomenológico que se ha elaborado hasta este momento. En ella encontramos la razón que nos lleva a conectar eventos similares, acoplados a un

\footnotetext{
${ }^{3}$ Ver, por ejemplo, Vaught (1986), en donde se argumenta la necesidad de incluir una "Cuarteridad".

4 "By the phaneron I mean the collective total of all that is in any way or in any sense present to the mind, quite regardless of whether it corresponds to any real thing or not" (Peirce 1931, p. 1284), escrita en 1905. Interpreto real thing como existencia fisica porque en 1905 Peirce ya había desarrollado su modo de realismo, por lo que hablar de algo que no es real y aun así tiene efectos en la mente es un sinsentido.
} 
principio general; y también encontramos la base de la posibilidad de formar hábitos. La Representación se manifiesta en las intenciones y en los significados. Como tal, y a diferencia de la Segundidad, no reaccionan directamente en la materia. Su efecto es producido con un impulso distinto, presentándose como una mediación entre otras dos cosas.

A lo largo de sus consideraciones fenomenológicas, es evidente que la noción de phaneron no es suficiente para lo que Peirce quiere decir. A pesar de haber comenzado como "algo que está presente frente a la mente", la fenomenología se comienza a expresar en términos de modos de ser. Cuando la metafísica comienza a aparecer, encontramos que los escritos abandonan cada vez más los términos antiguos (cualidad, posibilidad de sensación, fuerza, reacción, representación) y empiezan a favorecer los términos máximamente abstractos y generales: Firstness, Secondness y Thirdness. La Primeridad es "el modo de ser que es como es positivamente, sin consideración a nada más". La Segundidad es "el modo de ser con respecto a un segundo, pero sin consideración a un tercero". La Terceridad es "el modo de ser que es como es al relacionar un segundo y un tercero". Esta enunciación de las categorías se encuentra en CP 8328, pero Peirce ensayó decenas de definiciones a lo largo de sus manuscritos inéditos y de su correspondencia.

\section{Vaguedad y hecceidad}

La sensación pura de la Primeridad, la fuerza de la Segundidad y la mediación de la Terceridad encuentran instanciaciones en terrenos repletos de fenómenos. Esto es lo que Peirce quiere decir cuando califica a las categorías como tonos de pensamiento: momentos indefinidos de relación con lo que él nos ofrece como realidad. Cada categoría, sin embargo, es indefinible como un sólo fenómeno, pues agrupa una serie de instanciaciones heterogéneas. Cada una de estas instanciaciones tiene independencia conceptual, pero la línea que se tiende entre ellas (la categoría) revela un tono común. La navegación entre las diferentes caras de cada categoría en este sentido (es decir, analizando la individualidad de cada concepto a la luz de las resonancias comunes entre ellos) es un trabajo que aún debe de desarrollarse en profundidad. Un listado parcial de algunas estas instanciaciones (hábito, indeterminación o lenguaje, para la Terceridad; mecanismo, identidad o determinación para la Segundidad) hace entrever algunos conceptos tan fundamentales como problemáticos para la Biología contemporánea. Para comenzar a mostrar la conexión con las ciencias de la vida, en esta sección se explora la realidad la Terceridad y de su relación, en ocasiones tensa, con la Segundidad. Para ello, sigo el énfasis que da Richard Rorty (1961) a la Terceridad como vaguedad.

Podríamos preguntarnos cómo es que algo puede ser realmente vago. La experiencia inmediata, este paso constante a través de singularidades, parece estar permeada por entidades completamente determinadas, en el sentido de "estar aquí y ahora" También parece estar completamente determinada en el sentido de considerar a la realidad como un conjunto de entidades cuyas características ya están dadas; cualquier falla o indeterminación en nuestras descripciones se debe a una medida de ignorancia o a una incompetencia lingüística. Esta visión de una realidad absolutamente determinada por este tipo de existencia, por el "aquí y ahora" que nombramos más arriba con el término de hecceidad, es para Peirce la dimensión más inmediata y de más fácil comprensión, pues es la centrada en la actualidad: en la explicación de lo real por medio de hechos brutos. Los sistemas metafísicos que sólo aceptan la Segundidad son aquellos a los que "les gustaría explicar todo por medio de fuerza mecánica" (Peirce 1992e, p. 164). En estos sistemas podemos reconocer los intentos de explicación científica completamente fisicalista, que anima que un recuento satisfactorio de cualquier fenómeno puede ser reducible a la interacción, a través de la fuerza ciega (y, en tanto que actual, absolutamente determinada) ${ }^{5}$ entre las partes

${ }^{5}$ Cuando Peirce habla de indeterminación, está pensando en dos afluentes específicos. Por un lado, la indeterminación en la que no están especificadas absolutamente todas las características del objeto; por el otro, la incapacidad de decir el lugar exacto y tiempo instantáneo en que 
que lo componen.

Parecería que podemos describir el universo a través de una visión absolutamente centrada en Segundidad. Entonces, ¿por qué, para Peirce, esta visión de la realidad es incompleta? Podemos parafrasear la máxima pragmática: aquello que es real es aquello que tiene efectos y la totalidad de la realidad es la suma de estos efectos. En el sistema de categorías de Peirce, hay otros eventos, otras entidades, que pueden tener un efecto y que no están dictadas por acción y reacción mecánica. Un ejemplo sacado de la semiótica puede ayudar a entender esta postura. Cuando un general da la orden a sus soldados de bajar las armas, dicha orden causa un efecto en el comportamiento; más aún, ese efecto es repetible. Thomas S. Short ofrece un ejemplo zoológico: un aroma de madera podrida puede producir el efecto en un animal insectívoro de rascar la madera para encontrar larvas (Short 2009, pp. 159-161). A pesar de que en ambos casos el segundo evento (obedecer la orden; rascar un tronco caído) puede caracterizarse como respuesta del primero (el grito de la orden; el olor de madera podrida), difícilmente se puede dar un recuento de esta relación en términos de acción-reacción puramente física.

Hay varias críticas que se pueden realizar frente a estos ejemplos, pero sólo mencionaré dos que considero relevantes en este momento. En primer lugar, se podría criticar que estos efectos son producto de una relación no sólo contingente, sino incluso arbitraria. De acuerdo: pero eso es precisamente el punto que le interesa a Peirce, y que lo distingue de la Segundidad y la visión mecanística que esta categoría nos ofrece. En efecto: cada interpretante semiótico y cada relación de signos (y cada instanciación de Terceridad) está rodeada de elementos que no tienen una conexión lógica entre sí (cf. May 2005, p. 51). O, mejor dicho y siguiendo la sugerencia del propio Peirce: tienen una conexión lógica, pero es un tipo distinto de lógica. Es una lógica centrada en la vaguedad. Desafortunadamente, aunque hay varios lugares en los que está insinuada, no hay ningún manuscrito en el que esta idea esté desarrollada en su totalidad. Por ejemplo, en "Issues of Pragmaticism" (Peirce 1992f) comenta: "Logicians have been at fault in giving Vagueness the go-by, so far as not even to analyze it. The present writer [...] can here only give a definition or two with some proposals respecting terminology" (Peirce 1992f, p. 350) y a continuación siguen un par de páginas en donde Peirce elabora brevemente la distinción entre precisión y determinación, sugiriendo una estructura conceptual más amplia. A pesar de la aparente falta de conexión lógica, no es posible pensar que la Terceridad está absolutamente indeterminada. Para ser una mediación entre hechos ciegos, realizada a través de hábitos, debe de haber algo en la Terceridad que nos permita agrupar distintas instancias particulares en un tipo general. Es decir, hay una tensión (o tal vez sea mejor decir un equilibrio) entre la contingencia de las relaciones triádicas y el elemento que organiza a las instancias particulares como ejemplos reconocibles de un hábito o una ley.

En la segunda crítica, podríamos decir que ambos ejemplos están teñidos de intencionalidad y, peor aún, de mentalismo. De nuevo, de acuerdo: pero tanto el ejemplo de Peirce como el de Short quieren expresar algunas características de la Terceridad que podemos utilizar por analogía. La intencionalidad es sólo una de las manifestaciones de la categoría de Terceridad, que también cuenta entre sus filas a los hábitos, las leyes y los comportamientos legiformes, las intenciones, los signos, el lenguaje, la interpretación, la representación, la libertad. Todas estas concepciones son, para Peirce, fenómenos reales que a través del modus operandi de la Terceridad son considerables como principios activos de la realidad. "Es cierto", nos dice Rorty (1961) que todas estas instancias sólo se parecen entre sí en el hecho de ser triádicas. Pero al exhibir más y más las maneras en las que la Terceridad aparece, aumenta nuestro entendimiento de cualquier Tercero (i.e., elemento de la categoría de Terceridad).

Algo que se desprende de ambas críticas (y de su respuesta) es que cada instanciación de Terceridad tiene en sí una inevitable vaguedad. Los "Terceros" son un equilibrio entre la arbitrariedad y la determinación, y poseen un carácter temporal (en oposición al carácter inmediato e instantáneo de la

algo ocurre, en contra de la hecceidad. Ambas indeterminaciones son parte constitutiva de la Terceridad, y se pueden tratar bajo la misma idea de indeterminación o vaguedad. 
Segundidad). Dicho de otra forma: la Terceridad comprende los elementos reales del mundo cuya determinación no es completa. Un hábito, por ejemplo, tiene efectos en la realidad; pero es imposible reducir la descripción del hábito en sí a una descripción mecanística, porque un hábito cualquiera tiene un número de maneras indeterminado e indeterminable de actualizarse. Es por esto que una de las caras de la Terceridad es el concepto que Peirce llamaba alternativamente generalidad, indeterminación o vaguedad. La creencia en la realidad de esta doble indeterminación (i.e., la realidad de elementos fuera del hic et nunc de la hecceidad, y la presencia de características vagas en estos elementos) forma el núcleo de lo que Peirce llama su doctrina realismo escolástico.

He aquí una definición tentativa de la concepción compleja de vaguedad: aquel estado positivamente general que une a una serie de estados singulares bajo un mismo tipo. Estos estados singulares forman un continuo perfecto en tanto que agrupación, debido a que los que los une en el grupo es precisamente la indeterminación de alguna de sus características. Cualquier instancia de Terceridad contiene en sí la vaguedad. Consideremos cualquier signo; por ejemplo la palabra "verde". En su indeterminación, el interpretante con el que se asocia (es decir, el efecto que de hecho causa en una persona que lee esa palabra) ofrece un abanico continuo de tonalidades y brillos del color verde. El hábito que ocasiona una relación de la palabra con su interpretante es real, y por lo tanto, hay un elemento ontológico en nuestro universo que trae consigo, como parte inseparable de sí, la indeterminación. La consecuencia directa de esto, que tendrá que esperar su propio espacio para ser desarrollada satisfactoriamente, es que queda automáticamente desarmado el lugar común de la ciencia positivista que reza que cualquier indeterminismo es la medida de nuestra ignorancia -que es una posición nominalista por excelencia-. Baste señalar que la salida ante este modo del optimismo científico no se lleva a cabo apelando al azar, sino a la realidad de la vaguedad en el universo.

Prácticamente en todos los escritos de Peirce, bajo la superficie lingüística, epistemológica o incluso metodológica de sus discusiones, encontramos el mismo motor - la misma gran idea - que anima todas sus preocupaciones. Este motor es ontológico; es una manera de concebir la realidad. Es una visión que sale de las capacidades intelectuales humanas, porque es la herramienta que tenemos para comunicarnos con lo que nos rodea, pero en última instancia lo trasciende. Los fenómenos con los que nos comunicamos obligan a revisar los presupuestos metafísicos que nuestras capacidades o incapacidades parecen ofrecernos como los únicos posibles. Sólo si aceptamos este mensaje general del sistema de Peirce es posible ver el profundo efecto que tiene en nuestro acercamiento a los sistemas biológicos. La semiótica de Peirce, y la metafísica que la subyace, no es simplemente un apoyo para organizar el alud de datos que nos ha ofrecido el último siglo en cualquier área de la biología: es una estructura de pensamiento que nos permite rescatar de manera fundamental las distintas maneras de manifestarse de los objetos que nos proveen estos datos. Es decir, nos permite rescatar la característica de open-endedness que es fundamental en el funcionamiento de sistemas biológicos.

\section{La vida en la Terceridad}

Recientemente, la filosofía de Peirce ha tenido un resurgimiento y popularidad que nunca conoció en su época. En parte se debe a las reinterpretaciones biológicas, particularmente en el campo llamado biosemiótica. La labor de los investigadores de la biosemiótica es, la mayoría de las ocasiones, una naturalización de los procesos comunicativos de la vida en todos sus niveles, desde molecular hasta ecológico. ${ }^{6}$ La biosemiótica ha tratado, particularmente durante las últimas dos décadas, de mostrar una visión de la comunicación biológica como algo real, y que los códigos biológicos (por ejemplo, el código

\footnotetext{
${ }^{6}$ Para una revisión de las inquietudes biosemióticas, los problemas que plantean a la investigación biológica tradicional y una breve enumeración de estudios de caso particulares, ver Kull, Emmeche \& Favereau (2008).
} 
genético) no son simplemente una mera abreviatura o imagen poética de un proceso que nada tiene que ver, en realidad, con el proceso de significación.

A pesar de que el presente texto es cercano a la tradición de la biosemiótica (no es exagerado decir que sin la tradición biosemiótica no existiría), no es el interés primario mostrar una organización de la comunicación biológicos a través de la tríada semiótica de Peirce y dar cuenta de la comunicación como caso particular. Por otro lado, tampoco se inscribe por completo en el proyecto a gran escala de Peirce, su "cosmología evolutiva", en la que trata de dar un recuento del origen de las leyes físicas a través de un proceso de selección de leyes, en cierto sentido análogo a la teoría evolutiva darwiniana. Mi interés es más general y más acorde con el tono de la filosofía de Peirce, según la entiendo: explorar las consecuencias de la existencia real de las grandes categorías de dicho sistema dentro de nuestro entendimiento de los sistemas biológicos. Es decir, tomar como punto de partida la existencia de entidades reales que el discurso de la filosofía de la biología explícitamente niega o, en el mejor de los casos, simplemente ignora. Así, este texto es un eco de la advertencia constante de Peirce en contra del nominalismo, cuando aparece en los estudios biológicos; pues cuando el nominalismo ingresa a la biología desmonta la posibilidad de validar cualquier explicación que no intente reducir cualquier fenómeno dado en términos que no sean exclusivamente los asociados a la Segundidad: hechos brutos, fuerza ciega y una visión exclusivamente mecanicista de la vida. ${ }^{7}$ La gran diferencia es la diferencia entre la semiosis de Peirce y su sistema de categorías: la primera presupone al segundo, pero las categorías nos muestran que hay una serie de instancias además de la semiosis que deben de expresarse en términos de Terceridad.

Cuando los fenómenos biológicos se desean reducir, a toda costa, a los términos que prescribe una metafísica centrada en Segundidad, se pone en funcionamiento una cadena de normas y presupuestos. Cada elemento tiene que dar cuenta de su participación en las interacciones de las que toma parte, y la manera en que lo hace es a través de un listado finito de sus características físicas. La manifestación de estas características es completamente fisicalista. La manera en que se comporta cada elemento es previamente determinable por completo y, frente a otro elemento cualquiera, sólo basta un poder de cálculo suficientemente grande para determinar los resultados posibles. Daniel J. Nicholson lo expresa de la siguiente manera, como uno de los principios básicos del mecanicismo biológico: "Biological wholes are directly determined by the activities and interactions of their component parts, and consequently all properties of organisms can be characterized from the bottom up" (Nicholson 2012, p. 153). En última instancia, esta visión afirma que un fenómeno biológico puede y debe reducirse a interacciones físicas puras derivadas de los atributos físicos de cada uno de sus elementos.

Muchos conceptos biológicos llegan así a una especie de impasse. A pesar de un esfuerzo por matematizar las descripciones biológicas, visible clara y explícitamente desde los trabajos de la llamada Síntesis Moderna, la descripción de fenómenos biológicos consistentemente hace uso de términos e imágenes que han resistido su reducción a términos fisicalistas. En realidad, el problema no es tanto que los fenómenos en sí no se puedan describir propiamente como una serie de interacciones puramente mecánicas: de hecho, el popular campo llamado "nuevo mecanicismo" está centrado en este impulso de reducción. ${ }^{8}$ Sin embargo, y a pesar de la supuesta claridad explicativa de estos acercamientos, continúan llevándose a cabo discusiones y debates alrededor de términos básicos y de uso cotidiano. ¿Cómo podemos, por ejemplo, entender el uso de "función” en biología? ¿En qué términos es necesario fraguar las explicaciones en biología del desarrollo? ¿Debemos recurrir al concepto de clases naturales al hablar acerca de sistemas biológicos en distintos niveles? Podemos multiplicar las preguntas que aún no tienen respuesta, pero de alguna u otra manera, constantemente reaparece un espectro de finalidad en ellas. En una conocida cita, Susan Oyama (2000, p.31) se pregunta si estas discusiones están superadas; si no será una

\footnotetext{
${ }^{7}$ En este sentido, el practicante de biosemiótica que siento más cercano a las ideas presentadas aquí es Marcello Barbieri. Ver, por ejemplo, Barbieri (2002).

${ }^{8}$ Ver, por ejemplo, Machamer, Darden \& Craver (2000), cuyo número de citaciones en el momento de escribir el presente texto es de 1245.
} 
especie de metafórico "patear un caballo muerto", según la expresión inglesa. "Nada me gustaría más que dejar de patearlo", contesta, "pero cada vez que pienso que me libero de él, me regresa las patadas y hace cosas groseras en el medio intelectual y político. Sólo miren la cantidad de caballúnculos, infracaballos y metacaballos" que denuncia en su libro.

Cierto: el mundo biológico está poblado de caballos fantasma, pero la respuesta que podríamos dar, a la luz de la discusión anterior, es que esas presencias nocivas no son un subproducto del pensamiento biológico descarriado o de una persistencia de un ansia escolástica, que quiere interpretar absolutamente todo a la luz de un Primer Motor teológico; más bien se tienen que aceptar como parte natural de un lenguaje el cual es imposible trascender (Rorty 1961) y se tienen que canalizar para que tomen sus dimensiones y sus características apropiadas. Dicho de manera peirceana, las alucinaciones equinas de Oyama tienen su origen en las posiciones nominalistas, que llevan a pensar que los estados generales a los que apuntan no son vagos sino absolutamente determinados; i.e., que los estados generales se pueden describir en términos de singularidades. No puedo hacer suficiente énfasis en la lección de Peirce: que esta discusión, que aparentemente es una discusión bizantina lingüística o semántica, es en realidad una discusión ontológica. Aún si pudiéramos ingresar a los cimientos de la biología con las finas herramientas de Oyama para ajustar la manera de hablar, abandonar la terminología determinista y proponer términos nuevos, el determinismo sería inevitable en tanto se quiera reducir la Terceridad a términos de Segundidad. Esta confusión entre niveles es lo que lleva a un mismo autor a hablar de la vida en términos pasmosamente mecanicistas (Dawkins 1986) y, en el mismo texto, decir que cuando caen semillas de un árbol, "está lloviendo información [...] para hacer árboles" como si "llovieran discos de computadora" (Dawkins 1986, p. 158).

El concepto de información es, precisamente, el ejemplo concreto del presente texto. La información biológica es un concepto relativamente difuso, pero que permea todos los estratos de la actividad de las ciencias de la vida. Intuitivamente se puede aplicar a un rango enorme de eventos biológicos: una célula que se comunica con otra del mismo organismo a través de señales químicas, o de una célula de vida libre que detecta cambios en el medio; un animal que interactúa con su medio biótico o abiótico; cadenas de señalización en la que hay modificaciones terciarias o cuaternarias de proteínas que tienen un efecto regulativo. La concepción de información más difundida y tal vez más influyente es la información genética, que ha ocupado un lugar central en el discurso biológico desde hace poco más de medio siglo. La genética y la biología molecular han girado desde entonces alrededor de la concepción de que en el ADN, y el proceso de traducción del ordenamiento de los monómeros que lo componen en aminoácidos, se encuentra un fragmento clave para entender y describir los comportamientos de los sistemas biológicos en todas sus escalas.

Existen muchas razones por las cuales este fenómeno biológico se haya interpretado de la manera en que ocurrió. La elucidación de estos hechos fue realizada poco tiempo después del origen de una rama completa de estudio, la teoría de la información, en el estudio clásico de Claude Shannon (1948). La configuración secuencial de los ácidos nucleicos la posicionó como objeto de estudio perfecto para la teoría de Shannon, la cual analiza la comunicación de una secuencia de señales cualquiera desde una fuente a un receptor. Al dotar a cada base nitrogenada con la identidad de una señal discreta, el marco conceptual de Shannon fue transportado a las ciencias biológicas - hibridación que permitió incrementar la eficiencia de análisis de las secuencias y generar nuevas perspectivas en la naciente ciencia de la biología molecular.

¿Por qué esta convergencia y aplicación resulta problemática? Shannon hace énfasis en las frases finales de su artículo original: la teoría de la información sólo se puede utilizar para una comunicación literal de una serie de señales. No hay manera de representar una dimensión semántica a través del análisis de Shannon. Así, la información parece presentar dos caras: por un lado, hay un concepto validable de información, en el cual se puede introducir como contrabando otro que no tiene aplicación válida; i.e., la 
idea de que los genes contienen un elemento que informa la creación de proteínas y, a un mediano plazo, las funciones de los organismos en que los genes que las crean se expresan. La convergencia puede tratarse como un artefacto esencialmente histórico, en el que el surgimiento casi sincrónico de dos ramas importantes de investigación invistió de validez científica una serie de preconcepciones de carácter variado, tanto científico como filosófico o incluso religioso. En particular, parecen validar la idea de que el Mundo es un Texto apto para ser interpretado (el lado religioso de esta metáfora sería que el autor de dicho texto es una deidad). Posiblemente el trabajo que más elegantemente trata este tema, junto con sus consecuencias, es la obra de la historiadora de la ciencia Lily Kay (2000).

Hay otro argumento más cercano a nuestros intereses presentes: las consecuencias en el pensamiento científico si se toma a la información como una entidad explicativa en el estudio de los seres vivos. Esta visión es propuesta, en sus instancias más conocidas, por Sahotra Sarkar (2001), Paul E. Griffiths (2001) y Meter Godfrey-Smith (2000, 2007). El argumento es, a grandes rasgos, el siguiente: al tratar de confundir el uso de la información biológica con la aplicación de la teoría de Shannon, introducimos un elemento a una molécula de ADN que realmente no posee. Este elemento es una especie de semántica: una representación de la característica que se supone que informa. Este contrabando es pernicioso para el desarrollo de las ciencias biológicas, debido a que introduce un determinismo genético, que encuentra su expresión en la creencia de que las cadenas de ADN determinan completamente el desarrollo, morfología, funcionamiento e incluso comportamiento de los seres vivos. En resumen, estamos introduciendo una entidad inexistente (la información biológica) en nuestras explicaciones, que además parece tener un poder causal más grande que las entidades que sí existen.

Los argumentos del párrafo anterior sólo son válidos si estamos comprometidos con una visión del mundo en el que las únicas entidades existentes son los objetos físicamente localizables, y los únicos efectos que podemos aceptar son los que están derivados por atributos y actividades que éstos poseen. Dicho de otra manera, cuando estamos comprometidos con una visión del universo en el que la única categoría metafísica es la de determinación pura, i.e., Segundidad. Las únicas partes aceptables de las explicaciones son las que puedan ser atribuidas a causalidades mecánicas y relaciones lógicas entre los elementos que componen el objeto de estudio. Así, cualquier indeterminación en nuestros recuentos de los sistemas biológicos apunta a una sola causa: nuestra ignorancia de los mecanismos particulares que se esconden detrás de los efectos que observamos. Hago notar lo irónico de la solución: para evitar un determinismo genético (es decir, para contrarrestar una visión en que los estados generales a los que apunta una secuencia están particularmente determinados) es necesario abandonar todo indeterminismo ontológico (es decir, es necesario adoptar una visión en la que lo único que existe es la determinación mecanicista de las instancias particulares). Por lo tanto, los críticos citados arriba prescriben como cura el abandono de todo lenguaje con tintes semánticos en la biología. La única posible excepción es el llamado código genético, la relación uno-a-uno entre ácidos nucleicos y aminoácidos, debido a que "los genes especifican la secuencia de aminoácidos a través de un proceso de molde” (Godfrey-Smith 2007, p. 109).

Tal es el argumento fundamental del presente texto. Las críticas al concepto de la información biológica y el abandono del "lenguaje semántico" son una consecuencia directa de una visión ontológica absolutamente nominalista. Es imposible para los autores citados aceptar una biología fraguada con uso de términos de Terceridad simplemente porque no creen que ésta sea un elemento de la realidad, a pesar de que cotidianamente estos términos se usen explícita o implícitamente. La reducción de la realidad a términos exclusivamente de Segundidad, vía una prohibición lingüística, muestra un recuento empobrecido de una realidad que consistentemente se revela como creativa y se rebela contra estas restricciones. Los fenómenos de los sistemas biológicos muestran, una y otra vez, su carácter irreducible a un nominalismo.

Sin embargo, irreductibilidad no es igual a cisma, o a oposición. Una de las diferencias más importantes del presente texto con la biosemiótica es que no tengo la intención de proponer una imagen de los sistemas biológicos que sea exclusivamente semiótica, y ni siquiera exclusivamente basada en 
Terceridad. A pesar de que la mayoría de los principales actores de la tradición biosemiótica se conciben a sí mismos como una oposición al mecanicismo, esta es un movimiento contrario al que proponía Charles Peirce. En última instancia, el sistema filosófico que he presentado someramente aquí no es idealista; es decir que no depende únicamente de la Terceridad para poner en escena una ontología funcional. Peirce insiste a cada paso que, a pesar de la importancia de la Terceridad, no podemos olvidar la Segundidad como categoría fundamental del universo. Cada signo, cada hábito y cada ley tienen una instanciación física, particular y actual, una "réplica". Y así como la Terceridad logra agrupar instancias particulares a través de la realidad de sus tipos generales, las réplicas particulares son la manera de existir de la Terceridad. Decir que existe un hábito que nunca se actualiza en una instancia particular es decir algo que no tiene sentido.

A través de la vaguedad de la Terceridad, una vaguedad que tiene efectos en la realidad, es posible dar cuenta de lo apropiado de términos y maneras de pensar que fundamentan la estructura conceptual de la biología. Tanto el caso particular del concepto de información como las críticas a las que ha estado expuesto pueden observarse a la luz de esta articulación ontológica. No intento descalificar los análisis críticos del concepto de información anteriormente citados. Simplemente, intento mostrar que su punto de vista ontológico es nominalista: según ellos, aquello a lo que apunta la información (tanto el concepto "información" como la entidad "información), debería de ser localizable como una entidad particular y actualizada. Claramente no es el caso: el fin del hábito de cualquier información biológica es un fin no determinado en tanto que general. Es algo que escapa de nuestras manos cuando, a la manera mecanicista, tratamos de buscarlo en los atributos de un objeto físico; pero algo que, a final de cuentas, tiene efectos sobre las maneras de ser de los sistemas biológicos.

Para resumir y concluir, es posible apelar una última vez a Peirce, esta vez a su lógica argumentativa, la cual no fue mencionada en este texto. Peirce propone, como complemento de la deducción y de la inducción, el modo argumentativo de abducción, que es caracterizado por él como sigue: "The surprising fact, $\mathrm{C}$, is observed. But if $\mathrm{A}$ were true, $\mathrm{C}$ would be a matter of course. Hence, there is reason to suspect $\mathrm{A}$ is true" (Peirce 1931, p. 5.189).

Tras una sustitución con nuestros dos conceptos centrales (y un poco de paráfrasis), regresa a nosotros el punto central de este escrito:

- A pesar de las críticas al concepto de información, observamos continuamente sus efectos en los sistemas biológicos (y seguimos apelando a cualquiera de sus formas para explicarlos).

- Pero si la Terceridad fuera real, sería natural incluir a la información en nuestros recuentos ontológicos.

- Por lo tanto, tenemos razones para pensar que la Terceridad es un elemento importante de la realidad.

\section{Postscriptum}

A lo largo de este texto, intenté presentar en términos generales una serie de preocupaciones ontológicas como algo valioso. En lugar de presentar una visión restrictiva, en la que la ontología sea un mero listado a priori de los elementos que existen en la realidad, a partir de los cuales debemos construir un recuento de la realidad francamente poco interesante, la opción que nos presenta Peirce es una fuente primaria de creatividad, pues el mundo mismo que él intenta retratar es creativo y abierto. Dicha apertura está asegurada por la inclusión de la vaguedad, la cual abandona sus resonancias negativas y se establece como un elemento organizador para el entendimiento de fenómenos biológicos. Así, creo que se puede decir que las propuestas de este trabajo emergen de una serie de contrastes entre el discurso de la filosofía de la ciencia, la práctica y el discurso de las ciencias biológicas y lo adecuado o inadecuado de los presupuestos 
metafísicos que se manejan cotidianamente.

¿Estaría Peirce de acuerdo en esta lectura de su obra? Creo que sería imposible dar una respuesta unívoca. Lo más probable es que por una u otra razón la calificaría de nominalista, pues lanza este epíteto incluso contra algunas de sus más grandes influencias o almas filosóficamente emparentadas (por ejemplo, Kant, Duns Scotus o William James). Sin embargo, el objetivo no era hacer una exégesis del sistema completo de Peirce, sino evaluar la relevancia de ciertos conceptos aislados frente a problemas biológicos. Lo realizado aquí es, entonces, un intento por extirpar un grupo pequeño de conceptos que conviene a las ideas biológicas que se tocan (i.e., Terceridad, vaguedad, hecceidad) mientras se trata de hacer justicia a tales conceptos - no perder de vista su lugar en el intrincado sistema filosófico del que provienen-. Una de las principales dificultades de este ejercicio radica en que Peirce nunca fue explícito acerca de la capacidad de aplicar su sistema de manera tan expansiva. A pesar de que ciertos autores han negado la capacidad de construir un sistema semiótico que abarque una visión extensa de la biología -como por ejemplo, Short (2009, pp. 10-12) - que afirma que la semiótica y por extensión la Terceridad sólo debe de ser aplicable a humanos, y acaso a aquello que pueda entrar en la difusa categoría de comportamiento animal -es posible encontrar manuscritos y cartas con sugerencias de un uso mucho más extenso-. Por ejemplo, en una de sus últimas cartas (Peirce 1992e, p. 478) Peirce define al signo como algo que es determinado por un objeto y que determina un interpretante, el cual es "an effect upon a person"; inmediatamente después agrega "my insertion of 'upon a person' is a sop to Cerberus, because I despair of making my own broader conception understood". Así, su doctrina es definida en ocasiones como una pansemiosis: "this universe is perfused with signs, if not composed exclusively of signs" (Peirce 1931, p. 5448).

La pansemiosis ha sido criticada duramente como una posición antropomorfizante. De hecho, éste es uno de los bastiones críticos de Lily Kay (2000), al decir que la concepción escritural del genoma como un lenguaje (i.e., una Terceridad) natural, tiene ecos y de hecho es una continuación de la antigua cosmovisión en que el mundo es un texto interpretable. Es posible responder a Kay sin menospreciar la riqueza historiográfica de sus escritos. Por ejemplo, Bennett (2010) asevera que el antropomorfismo, extrañamente, funciona como un antídoto para la posición orgullosa que reserva ciertas actividades para los humanos y los coloca, de cierta manera, por encima del resto del universo. Aceptar que hay signos y otras maneras de Terceridad funcionando más allá de la mente humana desmonta una posición de antropocentrismo; y cuando este último desparece de la escena, es posible pensar un poco más libremente en la creatividad e indeterminación de modos de ser de cualquier entidad - lo cual es, desde mi punto de vista, uno de los más valiosos usos de la idea peirceana de vaguedad.

Bibliografía

Barbieri, M. (2002), "Has Biosemiotics Come of Age?”, Semiotica 139: 283-95.

Bennett, J. (2010), Vibrant Matter: A Political Ecology of Things, Durham: Duke University Press.

Bergson, H. ([1903] 2011), Introduction À La Métaphysique, Paris: Presses Universitaires de France.

Boler, J. (1980), “Peirce, Ockham and Scholastic Realism”, The Monist 63(3): 290-303.

Dawkins, R. (1986), The Blind Watchmaker, London: W.W. Norton and Co.

Godfrey-Smith, P. (2000), "Information, Arbitrariness and Selection: Comments on Maynard Smith", Philosophy of Science 67(2): 202-207.

Godfrey-Smith, P. (2007), "Information in Biology", en Hull, D. y M. Ruse (eds.), The Cambridge Companion to the Philosophy of Biology, Cambridge: Cambridge University Press, pp. 103-109. 
Griffiths, P.E. (2001), "Genetic Information: A Metaphor in Search of a Theory", Philosophy of Science 68(3): 394-412.

Haack, S. (1992), “Extreme Scholastic Realism': Its Relevance to Philosophy of Science Today”, Transactions of the Charles S. Peirce Society 28(1): 19-50.

Hausman, C.R. (1997), Charles S. Peirce's Evolutionary Philosophy, Cambridge: Cambridge University Press.

Kay, L. (2000), Who Wrote the Book of Life?: A History of the Genetic Code, Stanford: Stanford University Press.

Kull, K., Emmeche, C. y D. Favareau (2008), “Biosemiotic Questions”, Biosemiotics 1(1): 41-55.

Machamer, P., Darden, L. y C. Craver (2000), “Thinking about Mechanisms”, Philosophy of Science 67(1): 1-25.

May, T. (2005), Gilles Deleuze: An Introduction, Cambridge: Cambridge University Press.

Nicholson, D.J. (2012), "The Concept of Mechanism in Biology”, Studies in History and Philosophy of Biological and Biomedical Sciences 43: 152-63.

Oyama, S. (2000), The Ontogeny of Information: Developmental Systems and Evolution, Durham: Duke University Press.

Peirce, C.S. (1931), Collected Papers (8 Vols.), Cambridge, MA: Harvard University Press.

Peirce, C.S. (1992a), "What Pragmatism Is", en Peirce, C.S., The Essential Peirce: Selected Philosophical Writings (Vol. 1), Bloomington: Indiana University Press, pp. 331-345.

Peirce, C.S. (1992b), “A New List of Categories”, en Peirce, C.S., The Essential Peirce: Selected Philosophical Writings (Vol. 1), Bloomington: Indiana University Press, pp. 1-10.

Peirce, C.S. (1992c), "Seven Systems of Metaphysics”, en Peirce, C.S., The Essential Peirce: Selected Philosophical Writings (Vol. 1), Bloomington: Indiana University Press, pp. 179-195.

Peirce, C.S. (1992d), "A Guess at the Riddle”, en Peirce, C.S., The Essential Peirce: Selected Philosophical Writings (Vol. 1), Bloomington: Indiana University Press, pp. 243-279.

Peirce, C.S. (1992e), The Essential Peirce: Selected Philosophical Writings (2 Vols.), Bloomington: Indiana University Press.

Peirce, C.S. (1992f), "Issues of Pragmaticism”, en Peirce, C.S., The Essential Peirce: Selected Philosophical Writings (Vol. 1), Bloomington: Indiana University Press, pp. 346-359.

Rorty, R. (1961), “Pragmatism, Categories and Language”, Philosophical Review 70(2): 197-223.

Sarkar, S. (2001), "Biological Information: A Skeptical Look at Some Central Dogmas of Molecular Biology", en Sarkar, S. (ed.), The Philosophy and History of Molecular Biology: New Perspectives, Dordrecht: Kluwer, pp. 187-232.

Shannon, C. (1948), "A Mathematical Theory of Communication”, The Bell System Technical Journal 27: 379-423.

Short, T.L. (2009), Peirce's Theory of Signs, Cambridge: Cambridge University Press.

Vaught, C. (1986), "Semiotics and the Problem of Analogy: A Critique of Peirce's Theory of Categories", Transactions of the Charles S. Peirce Society 22(3): 311-26. 